\title{
Role of Diabetic Dyslipidemia on Coronary Atherosclerotic Severity in Acute Coronary Syndrome
}

\author{
Golam Mahfuz Rabbani ${ }^{1}$, Afzalur Rahman², Anisur Rahman Khan ${ }^{3}$, Nur Hossain ${ }^{4}$ Muhammad Badrul Alam \\ Amiruzzaman Khan ${ }^{6}$, Khondoker Asaduzzaman ${ }^{7}$
}

\begin{abstract}
:
Aims: To evaluate the association of coronary atherosclerotic severity in diabetic dyslipidemic patients of acute coronary syndrome.

Methods: This was a cross sectional comparative analytical study, done in the Department of Cardiology, Sir Salimullah Medical College Mitford Hospital and Ibrahim Cardiac Hospital \& Research Institute, Dhaka, during September 2009 to August 2010.
\end{abstract}

Results: Most of the patients (57.5\%) were in the age range of 40-70 years. Atherosclerotic severity in diabetic
ACS patients was significantly higher with low HDL, high TC/HDL and high LDL/HDL ratio. Low HDL, high TC/HDL and high LDL/HDL ratio are indicators of the extent and severity of coronary artery disease. More frequent dyslipidemia in diabetic ACS patients were low HDL and it was about $54 \%$.

Conclusion: Atherosclerotic severity in diabetic acute coronary syndrome patients was significantly more in dyslipidemic group than non dyslipidemic group.

Keywords: Diabetic Dyslipidemia, Acute Coronary Syndrome, Coronary Atherosclerosis

(Bangladesh Heart Journal 2016; 31(2) : 65-69)

\section{Introduction:}

By the year 2025, there will be more than 300 million type 2 diabetes sufferer worldwide. This epidemic will be followed by a wave of cardiovascular disease. As because, diabetes is in fact a serious vascular disease with poor prognosis. ${ }^{1}$

1. Junior Consultant, Department of Cardiology, Sir Salimullah Medical College and Mitford Hospital, Dhaka, Bangladesh.

2. Director and Professor, National Institute of Cardiovascular Diseases (NICVD), Dhaka, Bangladesh.

3. Associate Professor, Department of Cardiology, Mugda Medical College, Dhaka, Bangladesh.

4. Professor of Cardiology, Medinova Medical Services, Dhaka, Bangladesh.

5. Professor, Department of Cardiology, Sir Salimullah Medical College, Dhaka, Bangladesh.

6. Associate Professor, Department of Cardiology, Sir Salimullah Medical College, Dhaka, Bangladesh.

7. Assistant Professor, Department of Cardiology, Sir Salimullah Medical College, Dhaka, Bangladesh.

Address of Correspondence: Dr. Khondoker Asaduzzaman, Assistant Professor, Department of Cardiology, Sir Salimullah Medical College, Dhaka, Bangladesh. E-mail: asadareen@yahoo.com
Acute coronary syndrome (ACS) is one of the lifethreatening manifestations of coronary artery disease, includes from unstable angina, acute myocardial infarction (non-ST elevation and ST elevation) and sudden cardiac death. ${ }^{2}$

Diabeticpatients presenting with an acute coronary syndrome are morelikely to have a larger culprit lesion, higher incidence of multivessel disease and left main stemdisease (Morgan et al, 2004). Dyslipidemia is an abnormal amount of lipidse.g. total cholesterol e" 200 $\mathrm{mg} / \mathrm{dl}$ and/or LDL-cholesterol e" $130 \mathrm{mg} / \mathrm{dl}$ and/or HDLcholesterol d" $36 \mathrm{mg} / \mathrm{dl}$ and/or triglyceride e" $150 \mathrm{mg} / \mathrm{dl}$ ) in the blood. ${ }^{3}$

The relationship between coronary artery disease (CAD) and diabetic dyslipidemia in acute coronary syndromes has been rarely demonstrated in clinical and epidemiological studies. The extent of CAD in subtypes of patients with DM is unknown. ${ }^{2}$

As morbidity and mortality of diabetic ACS patient is more due to severity of coronary atherosclerosis, so we set out 
to examine if patients with dyslipidemia have more severe CAD when compared to nondyslipidemic patients.

\section{Patients and Methods:}

This was a cross sectional comparative analytical study, done in the Department of Cardiology, Sir Salimullah Medical College Mitford Hospital and Ibrahim Cardiac Hospital \& Research Institute, Dhaka during September 2009 to August 2010. All diabetic acute coronary syndrome patients admitted in those hospitals during the specified period were included in the study considering the inclusion and exclusion criteria.

The diagnosis of ACS was made in patients who presented with retrosternal chest pain of 20 minutes or more with electrocardiographic changes ( $T$ wave inversion $>1.0 \mathrm{~mm}$, ST-segment depression $>1 \mathrm{~mm}$ and or ST-segment elevation $>1 \mathrm{~mm}$ ) or Troponin-I levels $>0.06 \mathrm{ng} / \mathrm{ml}$.

Type 2 diabetes was diagnosed by taking documented past history of DM, RBS $>11.1 \mathrm{mmol} / \mathrm{L}$ or FBS $>7 \mathrm{mmol} /$ $\mathrm{L}$ or $\mathrm{HbA} 1 \mathrm{c}$ was $>7 \mathrm{mmol} / \mathrm{L}$. Serum lipid profile (total holesterol, LDL-cholesterol, HDL-cholesterol, and triglyceride) was measured as early as possible (within 24 hours).

When serum lipid profile showed total cholesterol $>200$ $\mathrm{mg} / \mathrm{dl}$ and/or LDL-cholesterol > $130 \mathrm{mg} / \mathrm{dl}$ and/or HDLcholesterol < $36 \mathrm{mg} / \mathrm{dl}$ and/or triglyceride > $150 \mathrm{mg} / \mathrm{dl}$ then they were considered as dyslipidemic patients and if total cholesterol d" $200 \mathrm{mg} / \mathrm{dl}$, LDL-Cholesterol d" 130 $\mathrm{mg} / \mathrm{dl}$, HDL-Cholesterol e"36 mg/dl and triglyceride d"150 $\mathrm{mg} / \mathrm{dl}$ then they were considered as nondyslipidemic patients.

All patients underwent coronary angiography. Two criteria were considered for coronary artery disease : percentage of obstruction and number of vessels affected. Where the main

artery lesion were scored as for the presence of lesions $(>50 \%$ ) in the proximal-3, middle -2 and distal segment1 ; as well as for the degree of obstruction $(<50 \%=1 ; 50-$ $70 \%=2 ;>70 \%=3)$. The secondary artery lesion were scored only as for the degree of obstruction $(50-70 \%=1$; $>70 \%=2$ ). Score was also divided into mild (when score $<9$ ), moderate (when score 9 to 17) and severe (when score >17).

\section{Statistical Analysis:}

Data were analyzed by the SPSS version 11.5 software program. Confidence interval was set at $95 \%$ level. Results were considered to be statistically significant if $p$ value was $<0.05$, very significant if $p$ value was $<0.01$, and highly significant when $p$ value was $<0.001$.

\section{Results:}

The study was done in Cardiology Department of Sir Salimullah Medical College and Mitford Hospital, Dhaka, with the collaboration of Ibrahim Cardiac Hospital \& Research Institute, Dhaka, during the period of September 2009 to August 2010. This study was carried out to identify the association of dyslipidemia with severity of coronary atherosclerosis in diabetic acute coronary syndrome patients.

A total of 123 patients were included in this study. Out of 123 patients 31 were non-dyslipidemic and 92 were dyslipidemic diabetic ACS patients. There was no significant difference $(p=0.06)$ between the mean age of dyslipidemic (57 years) and nondyslipidemic (53 years) patients. No significant difference $(p=0.104)$ in BMI was observed between this two groups.

Normal epicardial coronary artery and single vessel disease (SVD) were more in nondyslipidemic patients compared to dyslipidemic patients $(22.6 \%$ vs $0 \%$ and $64.5 \%$ vs $14.1 \%$ ). On other hand double vessel disease (DVD) and triple vessel disease (TVD) were more in dyslipidemic patients group compared to nondyslipidemic patients group. So, the difference between the two group was highly significant $(p=0.0001)$.

Type A, type B and type $C$ lesions (all types) were more in dyslipidemic patients compared to nondyslipidemic patients ( $55.4 \%, 76.1 \%, 51.1 \%$ vs. $48.4 \%, 35.5 \%, 22.6 \%$ ). However, type A lesion was not significantly different between two groups $(p=0.496)$ but type $B$ lesion and type $C$ lesion were highly significant between the two groups $(p=0.0001, p=0.006)$.

Mean percentage of stenosis in three main coronary arteries (LAD, RCA, and LCX) were significantly higher in dyslipidemic group than that of nondyslipidemic group $(p=0.0001, p=0.01$ and $p=0.0001)$. No left main (LM) disease found in nondyslipidemic patients but $12 \%$ dyslipidemic patients had LM disease, So the difference was significant $(p<0.05)$. LAD, LCX \& RCA lesions were significantly more in dyslipidemic compared to nondyslipidemic patients $(p<0.05)$. The difference were highly significant especially in LAD and RCA.

Table- II shows that mild score was more in nondyslipidemic patients compared to dyslipidemic patients $(90.3 \%$ vs. $7.6 \%)$; while moderate and severe score were more in dyslipidemic compared to 
nondyslipidemic patients group $(65.2 \%$ vs. $6.5 \%$ and $27.2 \%$ vs. $3.2 \%$ ). So the difference between the two group was highly significant $(p=0.0001)$.

The above table (III) showed that there was negative correlation between atherosclerotic lesion score and HDL cholesterol ("r" $=-.557$ and $p=0.0001$ ), so this correlation is highly significant. Atherosclerotic lesion score and TC/HDL ratio had positive correlation ("r"= .444 and $p=0.0001$ ) also the same positive correlation was existed between atherosclerotic lesion score and LDL/ HDL ratio ("r" $=.395$ and $p=0.0001$ ). So, $\mathrm{HDL}$
cholesterol,TC/HDL ratio and LDL/HDL ratio were highly significant. But other lipid fractions had no significant correlation.

The risk of severe atherosclerotic disease was higher in dyslipidemic patients than nondyslipidemic patients, as Odds ratio was $40.76(>1)$. So, dyslipidemia was significant risk factor for severe atherosclerotic disease.

The risk of multivessel disease was higher in dyslipidemic patients than nondyslipidemic patients, as Odds ratio was $41.01(>1)$. So, dyslipidemia was significant risk factor for multivessel disease

Table-I

Atherosclerosis lesion score between non-dyslipidemic and dyslipidemic patients

\begin{tabular}{lcccc}
\hline Parameters & $\begin{array}{c}\text { Non-dyslipidemic }(n=31) \\
\text { Mean } \pm \text { SD }\end{array}$ & $\begin{array}{c}\text { Dyslipidemic }(n=92) \\
\text { Mean } \pm S D\end{array}$ & t-value & $p$-value \\
\hline Lesion score & $4.81 \pm 4.08$ & $13.98 \pm 4.37$ & -10.277 & $0.0001^{\mathrm{s}}$ \\
\hline
\end{tabular}

Data were analysed by using student' $t$ ' test. The table (I) shows that atherosclerotic lesion score was more in dyslipidemic patients group than nondyslipidemic patients group (13.98 \pm 4.37 vs. $4.81 \pm 4.08)$, which was highly significant $(p=0.0001)$.

Table-II

Atherosclerosis lesion score severity between nondyslipidemic and dyslipidemic patients

\begin{tabular}{lcccc}
\hline Lesion Score & Nondyslipidemic & Dyslipidemic & $\chi^{2}-$ & \\
\hline Severity & $(\mathrm{n}=31)$ No. $(\%)$ & $(\mathrm{n}=92)$ No. $(\%)$ & value & p-value \\
Mild Score & $28(90.3)$ & $7(7.6)$ & & $0.0001^{\mathrm{s}}$ \\
Moderate Score & $2(6.5)$ & $60(65.2)$ & $77.926^{\mathrm{a}}$ & \\
Severe Score & $1(3.2)$ & $25(27.2)$ & & \\
\hline
\end{tabular}

Data were analyzed by using Chi-square $\left(\dot{\div}^{2}\right)$ Test; Mild Score $=$ Score $<9$. Moderate Score $=$ Score 9 to 17 . Severe Score $=$ Score $>17$.

Table-III

Correlation between different lipid component and atherosclerotic lesion score

\begin{tabular}{llll}
\hline Parameter & & CorrelationCo-efficient "r" & P value \\
\hline & TC & -.063 & $0.492^{\mathrm{ns}}$ \\
& HDL & -.557 & $0.0001^{\mathrm{s}}$ \\
& LDL & .004 & $0.963^{\mathrm{ns}}$ \\
Score & TG & -.010 & $0.917^{\mathrm{ns}}$ \\
& TC/HDL ratio & .444 & $0.0001^{\mathrm{s}}$ \\
& LDL/HDL ratio & .395 & $0.0001^{\mathrm{s}}$ \\
& NHDLC & .065 & $0.475^{\mathrm{ns}}$ \\
\hline
\end{tabular}




\section{Discussion:}

Diabetes is in epidemic proportion in our country and over half $(75 \%)$ of all diabetic patients die of coronary artery disease presenting as acute coronary syndrome. Tight glycemic control have benefit on microvascular disease but it show no benefit on macrovascular disease, such as coronary artery disease. ${ }^{4}$ Increased incidence of hypertension, insulin resistance and adverse lipid profile in diabetic patients predisposes to coronary artery disease. An atherogenic pattern of lipoprotein changes (low HDL, high TG and small size LDL) is often present for years prior to development of fasting hyperglycemia and diagnosis of type 2 diabetes mellitus. ${ }^{5}$ In the presence of hyperglycaemiaLDL becomes glycosylated and is poorly recognizedby the low density lipoprotein receptor. It is scavenged bytissue macrophages creating the foam cell, a constituent ofthe atherosclerotic plaque.So, lipid status is more important in CAD of diabetic patient. ${ }^{4}$

This cross-sectional analytical study revealed that dyslipidemia in diabetic patients were associated with higher risk of development of severe coronary atherosclerosis in terms of number of vessel involvement, type of lesions, percentage of obstruction and lesion score.

In this study 97 (78.86\%) patients were male and 26 $(21.14 \%)$ patients were female. Male female ratio was 3.73: 1. Female patients comprise a small part of the present study. In Bangladesh, almost all of the study reported an overwhelming majority of male patients. This study also showed that mean age of both group was around 56 years. Hochman et al, (1988) also showed that fewer women than men had myocardial infarction ( 36.6 percent vs. 47.6 percent, $P<0.001$ ) and their average age was 56.6

The analysis showed that most patients in this study population had dyslipidemia $74.8 \%$ (especially low HDL $53.6 \%)$. Nasir et al (2008) also showed that dyslipidemia in diabetic patient $76 \%$, which is almost similar to my study. ${ }^{7}$

In this study in general, dyslipidemic patients had more multivessel disease and complex type $B$ and type $C$ lesions. Left main disease was found only in dyslipidemic group. Morgan et al. showed that diabetic patients have a higher incidence of multivessel disease, left main stemdisease and complex type $\mathrm{C}$ lesion. ${ }^{4}$

In this study, the mean levels of lipid variables did allow the discrimination between the presence and absence of multivessel coronary artery disease. The analysis of
HDL showed that this variable decreased with the number of vessels affected. Patients with single-vessel disease had HDL levels of $40.65 \pm 8.51 \mathrm{md} / \mathrm{dl}$, whereas those with multivessel disease had levels of $31.21 \pm 9.28$ $\mathrm{md} / \mathrm{dl}(\mathrm{p}=0.0001)$. Several studies agree that low levels of $\mathrm{HDL}$ are correlated with the presence and severity of CAD. ${ }^{5}$ TC/HDL and LDL/HDL were higher in the multivessel groups when compared to the single vessel group (6.00 vs. 4.62 and 3.38 vs. 2.81). Similarly TC/HDL and $\mathrm{LDL} / \mathrm{HDL}$ were higher in multivessel coronary artery disease, showed by Penalvaet al. ${ }^{2}$

In addition, the analysis of severity of coronary artery disease using the score system ${ }^{8}$ showed that the variable HDL, TC/HDL and LDL/HDL were also significantly correlated with the atherosclerotic lesion score. HDL had negative correlation (inversely related) with atherosclerotic lesion score. TC/HDL and LDL/HDL had positive correlation with atherosclerotic lesion score. Similar relationship with low HDL shown by Murphy et al (2007) and positive correlation of TC/HDL and LDL/HDL with coronary lesion score were showed by Penalva et al (2008). ${ }^{5,2}$

Therefore, this finding reinforces the importance of the measurement of HDL, TC/HDL ratio and LDL/HDL ratio. They are indicators of extent and severity of the coronary artery disease. Penalva et al (2008) thus suggesting that the imbalance between TC and HDL levels plays a more important role in the pathophysiology of atherogenesis.

It is important to consider that the atheroprotective function of HDL is not restricted to reverse cholesterol transport, but can also transport antioxidant enzymes, break down oxidized lipid fractions, and neutralize their proinflamatory effects. ${ }^{9}$

Patients of diabetic acute coronary syndrome was my study population and low HDL, high TC/HDL and LDL/ HDL ratio were the markers of severe coronary artery disease. Fresco et al. reported that lowering lipid level occur 24 hours after an attack of acute coronary syndrome.${ }^{10}$ So it is important to measure lipid profile at admission to know the exact value and identify patients of higher potential risk.

\section{Conclusion and Recommendation:}

Atherosclerotic severity in diabetic acute coronary syndrome (ACS) patients were significantly more in dyslipidemic group.

The findings of this study emphasize the need for assessment of the lipid profile of diabetic ACS patients at admission, so to identify patients of higher potential risk. 
Multicentred broad based study in Bangladesh will help to support the findings and observations of this study.

\section{References:}

1. Taskinen, MR. Diabetic dyslipidemia. Atheroscler Sup 2002;3(1): 47-51.

2. Penalva, R. A., Marçal, O. H., Luis, C. L. C., Gilson, S. F., Ana, M. T. L. Lipid profile and severity of atherosclerotic disease in acute coronary syndrome. Arq Bras Cardiol 2008;90 (1):24-9.

3. NCEP. National Cholesterol Education Program (NCEP) Expend panel on Detection, evaluation and treatment of high blood cholesterol in adults (Adult Treatment panel III). Third report of the National Cholesterol Education Program (NCEP) expert panel on defection, evaluation and treatment of high blood cholesterol in adults (Adult treatment panel III) final report, Circulation 2002;106:3143-421.

4. Morgan, K. P., Kapur, A. Beatt, K. J. Anatomy of coronary disease in diabetic patients: an explanation for poorer outcomes after percutaneous coronary intervention and potential target for intervention. Heart 2004;90(1):732-8.

5. Murphy, C. J., Brunner, N. W., Taylor, C. M., Fox, R. S., Sundar, M. R., Penn, I. M., Ramanathan. Diffuseness of coronary arteriosclerosis in dyslipidemic diabetics versus nondyslipidemic diabetics. Canadian Cardiovascular Congress 2007;5 (9): 0509.

6. Hochman, J. S., Phillips, W.J. \&Ruggieri, D. The distribution of atherosclerotic lesions in the coronary arterial tree: relation to cardiac risk factors. Am Heart J1988; 116 (1): 1217-22.

7. Nasir, A., Jahangir, K., Tahir, S. S. Frequency of dyslipidemia in type 2 diabetes mellitus in patients of Hazara division. J Ayub Med Coll Abbottabad 2008; 20 (2): 51-4.

8. Nikkita, E., Viikinkoski, P., Valle, M. Prevention of progression of coronary atherosclerosis by treatment of hiperlipidaemia: a seven year prospective angiographic study. Br Med J1984;289 (1): 220-3.

9. Libby, P., Ridker, P. \& Maseri, A. Inflammation and atherosclerosis. Circulation 2002; 105 (1): 1135-43.

10. Fresco, C., Maggioni, A. P., Signorini, S., Merlini, P. A., Mocarelli, P. Fabbri, G. Variations in lipoprotein levels after myocardial infarction and unstable angina: the latin trial. Ital Heart J2002; 3 (1): 587-92. 